\title{
PD-L1 expression levels should be considered when identifying risk factors in NSCLC patients treated with nivolumab
}

\author{
Pengfei $\mathrm{Li}^{1}$ · Guowei Che ${ }^{2}$
}

Received: 24 July 2019 / Accepted: 27 November 2019 / Published online: 5 December 2019

(c) Springer-Verlag GmbH Germany, part of Springer Nature 2019

\section{Dear Editors,}

We read with great interest the article recently published in your journal by Bonaventura and colleagues [1]. The article evaluated serum proprotein convertase subtilisin/kexin type 9 (PCSK9) in 44 elderly patients with advanced non-smallcell lung cancer (NSCLC) under nivolumab treatment and finally proved that circulating levels of PCSK $9<95 \mathrm{ng} / \mathrm{mL}$ at the second cycle of nivolumab treatment served as an independent predictor for a better overall survival in elderly patients with advanced, pre-treated NSCLC. The authors have accomplished an excellent job; however, several questions must be answered before this conclusion can be drawn.

A limited number of 44 cases was included in the analysis only four variables including age, sex, smoking habit, and previous treatments were considered as potential confounders in the cox proportional hazards multivariate model. Indeed, age, sex, smoking habit, and previous treatments are risk factors which may exert substantial influence on patients' survival and there is no doubt these variables should be included in the analysis. This subset of 44 patients was enrolled in a previous study [2] in which patients received different lines of treatment. Thus, the authors should present a detailed description of the patients' prior treatment since it may have substantial influence on their survival.

Antibodies that block the programmed cell death-1 (PD1)/programmed cell death ligand-1 (PD-L1) pathway offer a promising approach for the treatment of advanced NSCLC.

This comment refers to the article available at https://doi. org/10.1007/s00262-019-02367-z.

Guowei Che

cheguowei_hx@aliyun.com

1 The Second Affiliated Hospital of Xi' an Jiao Tong University, Xi' an 710004, China

2 West China Hospital, Sichuan University, No. 37, Guoxuexiang, Chengdua 610000, China
Most of the published studies report poorer prognosis for patients with high PD-L1 expression, and that these patients have the most benefit when treated with anti-PD-1/PD-L1 antibodies [3]. Accordingly, it would benefit the study to consider the PD-L1 expression level in the cox proportional hazards model and compare the difference of PD-L1 expression level between the high and low PCSK9 groups.

\section{Compliance with ethical standards}

Conflict of interest The authors declare that they have no conflict of interest.

\section{References}

1. Bonaventura A, Grossi F, Carbone F et al (2019) Serum PCSK9 levels at the second nivolumab cycle predict overall survival in elderly patients with NSCLC: a pilot study. Cancer Immunol Immunother 68:1351-1358. https://doi.org/10.1007/s00262-01902367-z

2. Sarocchi M, Grossi F, Arboscello E et al (2018) Serial troponin for early detection of nivolumab cardiotoxicity in advanced nonsmall cell lung cancer patients. Oncologist 23:936-942. https:// doi.org/10.1634/theoncologist.2017-0452

3. Brody R, Zhang Y, Ballas M et al (2017) PD-L1 expression in advanced NSCLC: Insights into risk stratification and treatment selection from a systematic literature review. Lung Cancer 112:200-215. https://doi.org/10.1016/j.lungcan.2017.08.005

Publisher's Note Springer Nature remains neutral with regard to jurisdictional claims in published maps and institutional affiliations. 